\title{
Estrategia empresarial y tecnologías de la información en la gestión del conocimiento técnico-documental. Estudio del caso Nuclenor
}

\author{
Por Pedro Solana-González y Daniel Pérez-González
}

\begin{abstract}
Resumen: Experiencia y resultados de la implementación de un sistema de gestión de información técnico-documental en la empresa Nuclenor (Central Nuclear Santa María de Garoña). Con el propósito de compartir conocimientos y contribuir en la medida de lo posible a la difusión de este tipo de sistemas en otras empresas y organizaciones, se explica la estrategia organizativa y los objetivos definidos por Nuclenor para llevar a cabo este proyecto, y se analizan los requerimientos funcionales del sistema. Desde una perspectiva técnica se presenta la metodología utilizada para su realización y el modelo tecnológico de soporte. Por último se hace una revisión de los resultados y beneficios derivados de este proyecto para la empresa, y se comentan las líneas de investigación que están abiertas en este momento.
\end{abstract}

Palabras clave: Tecnologías de la información, Gestión documental, Sistemas de información integrados, Sistemas de colaboración.

Title: Enterprise strategy and information technologies in technical-documentary knowledge management. The case study of Nuclenor

Abstract: The experience and results of the development and implementation of a technical-documentary information management system in the enterprise Nuclenor (Power plant of Santa María of Garoña) are presented. In order to share knowledge and contribute as far as possible to the dissemination of this kind of systems in other enterprises and organizations, we explain the organizational strategy and objectives de functional requirements of the system. From a technical perspective the development methodology used and the support technological model of the system is presented. Finally, we review the results and benefits of the project for the enterprise, and comment the research lines currently open.

Keywords: Information technologies, Document management, Integrated information systems, Collaborative systems.

Solana-González, Pedro; Pérez-González, Daniel. "Estrategia empresarial y tecnologías de la información en la gestión del conocimiento técnico-documental. Estudio del caso Nuclenor". En: El profesional de la información, 2008, v. 17, n. 5, septiembre-octubre, pp. 487-501.

DOI: $10.3145 /$ epi.2008.sep.02

\section{Introducción}

Analizaremos la estrategia empresarial y las tecnologías de la información aplicadas en el desarrollo de un proyecto de gestión de información técnico-documental en la empresa Nuclenor. El esquema temporal

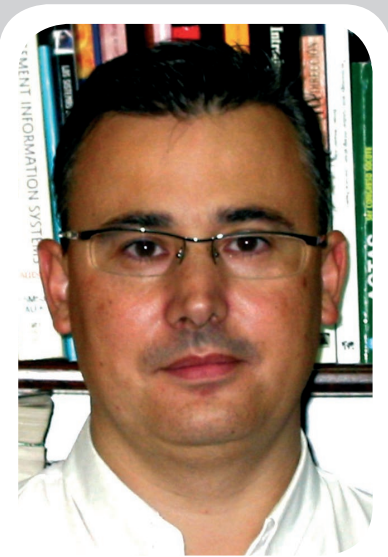

Pedro Solana-González, licenciado en informática por la Univ. Politécnica de Cataluña (UPC) y doctor ingeniero industrial por la Univ. de Cantabria (UC), es profesor de sistemas de información y diseño de bases de datos, y miembro del grupo $I+D+i$ de Informática de Gestión de la UC. Ha participado y dirigido proyectos de investigación para Nuclenor y para Solvay Química. Investiga en gestión documental, modelado y automatización de procesos de negocio (workflow) y cesos de negocio (workflow) y

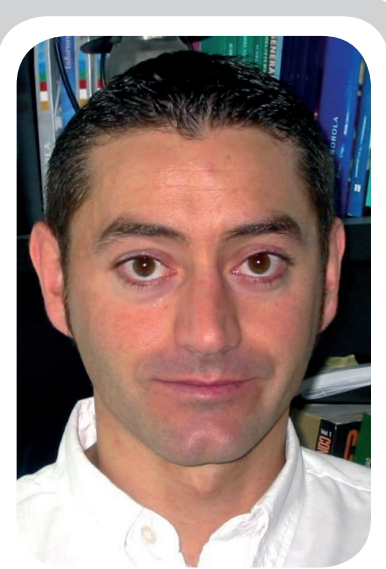

Daniel Pérez-González, licenciado y doctor en administración y dirección de empresas por la Univ. de Cantabria (UC), es profesor de sistemas de información y miembro del grupo $I+D+i$ de Informática de Gestión de la UC. Miembro de la Academia Europea de Dirección y Economía de la Empresa y colaborador del Institut for Market research of Kiel (Alemania). Investiga en sistemas de información organizativos, valoración de tecnologías de la información y gestión del conocimiento. del estudio abarca el periodo 2002-2004, en el cual se desarrolló e implantó el sistema, si bien con objeto de analizar el caso con una perspectiva mayor se comentan los antecedentes que permitieron a la empresa abordar este proyecto. 
El caso que se presenta tiene interés por cuanto se trata de una investigación aplicada en un contexto empresarial real -la empresa Nuclenor-, tanto por las particularidades específicas que tiene como caso único, al pertenecer a un sector como el nuclear, altamente regulado y sometido a rigurosos controles de calidad, como por las características y problemática común con otras organizaciones.

El análisis de este caso se plantea con el objetivo de compartir, más allá de los límites de la empresa y del sector industrial en el que se desarrolla, el conocimiento y las experiencias de Nuclenor en el diseño, desarrollo y puesta en marcha de su sistema de información técnico-documental. Para ello se expone en primer lugar la estrategia empresarial y objetivos del proyecto, en segundo lugar se analizan los requerimientos del sistema, para posteriormente explicar el modelo tecnológico de desarrollo, y finalizar con el análisis de los resultados y la exposición de las conclusiones más relevantes.

Los avances en el tratamiento de la información y en la gestión del conocimiento como soporte de los procesos de negocio, no sólo permiten hoy a las empresas que automaticen la gestión de sus datos, sino el ciclo de vida completo de la documentación -la generación, almacenamiento y catalogación de documentos, la distribución, compartición y uso (flujos documentales) de este conocimiento explícito ${ }^{1}$, así como su eliminación / conservación-. Este tipo de aplicaciones, llamadas de gestión documental, requieren realizar un análisis corporativo de los datos y documentos, así como del software y del equipamiento hardware para su implantación.

A este hecho se añade un importante cambio de enfoque en las organizaciones, en las que las aplicaciones de gestión y los sistemas de tratamiento de la información están evolucionando desde una perspectiva departamental, en la que el objetivo es solucionar problemas propios de una determinada unidad de negocio o grupo, hacia la gestión integrada de la información y la compartición del conocimiento por toda la organización, lo que permite alcanzar un elevado grado de eficiencia en la gestión.

Nuclenor, consciente de este hecho y de la importancia que tiene la calidad de la información y la eficiencia en la gestión de la documentación en el sector nuclear, tomó la iniciativa de definir los requerimientos funcionales y diseñar un sistema de gestión documental que ha evolucionado en los últimos años integrando la información de carácter técnico de la empresa: configuración de la planta (sistemas, zonas, equipos, etc.), gestión de trabajos de mantenimiento y experiencia operativa - know-how compartido que constituye la base para el aprendizaje organizacional y la difusión del conocimiento tácito (Marwick, 2001)-. De esta forma el sis- tema ha llegado a constituir un entorno de colaboración en base al conocimiento y a la gestión de los flujos de trabajo (workflow) técnico-documentales.

\section{"El sistema ha llegado a constituir un entorno de colaboración basado en la gestión del conocimiento y flujos de trabajo técnico-documentales"}

\section{Estrategia y objetivos}

Como empresa que opera en un entorno complejo fuertemente regulado y sometido a estrictos controles de seguridad y calidad, Nuclenor ha definido como un objetivo estratégico optimizar la gestión de la información técnico-documental de la Central Nuclear de Santa María de Garoña, y en este sentido, la dirección ha considerado el alto valor que tiene la información para la gestión de la empresa (Barranco, 2006).

\subsection{Antecedentes}

Basándose en esa estrategia se tomó la decisión de centralizar y unificar las aplicaciones de gestión de información de la empresa, que a mediados de la década de los noventa se restringían al ámbito de unas pocas secciones: Oficina Técnica de Ingeniería, Mantenimiento, y Garantía de Calidad. Para ello se realizó un análisis de la información de la organización (documentos, conocimiento técnico, listas maestras, etc.) y se identificaron los flujos de la documentación (d'AlòsMoner, 2006), al tiempo que se recogía la experiencia de las personas.

Un paso importante en esta línea se realizó con la especificación, desarrollo y puesta en marcha de un sistema de gestión documental, que estableció las bases para la evolución futura del sistema integrado de información técnico-documental, al que hace referencia este caso.

Los objetivos de éste fueron los siguientes:

- Acceso a la información: facilitar la consulta y acceso online a la documentación.

- Disponibilidad de información actualizada: controlar las modificaciones que se realizan sobre los documentos (revisiones), manteniendo la información siempre actualizada, evitando duplicidades y garantizando en todo momento el acceso a la documentación vigente.

- Automatización de flujos de trabajo: mecanizar los flujos de trabajo y el ciclo de vida de la docu- 
mentación, proporcionando una gestión más eficiente de las tareas diarias que desarrolla el personal de la empresa.

- Ahorro de espacio físico de archivo y reducción de la gestión del papel: disminuir el volumen de papel almacenado en los archivos de la empresa ahorrando espacio físico, y reducir el número de copias en papel y documentos en circulación mediante la distribución electrónica de documentos, facilitando su consulta online, e implantando nuevas formas de trabajo basadas en las tecnologías de la información.

Este proyecto precursor contó con la participación de la empresa $K E O N$, experta en la implantación de este tipo de sistemas, y con la colaboración de la Universidad de Cantabria, definida ésta a través de un acuerdo marco, que se plasmó en diversos proyectos de I+D.

Para el sistema de gestión documental se usó la plataforma Microsoft visual studio -con el lenguaje Visual basic 5.0-, y como gestor de bases de datos Informix online v. 7.31.UC5 sobre el sistema operativo HP-UX v. 10.2.

\subsection{El sistema integrado de información técnico-do- cumental}

La mejora de la calidad de la información y los flujos documentales logrados con el sistema de gestión documental, así como las sinergias alcanzadas con la coordinación de los trabajos de las diversas secciones, hacen que Nuclenor se plantee dar un paso más para optimizar la gestión de la información, con el diseño e implementación de un sistema de información técnico-documental, que ha supuesto un avance cualitativo en su estrategia de integración del conocimiento y de las aplicaciones de la empresa.

Nuclenor entiende como sistema integrado de información técnico-documental, en primer lugar, aquel capaz de interrelacionar de forma consistente en un modelo de datos único tanto las entidades que permiten la gestión de la información técnica, como las que permiten la gestión del ciclo de vida y flujos documentales de la empresa; y, en segundo lugar, considera que debe integrar las distintas aplicaciones técnicas y de gestión documental que existían en ese momento en la empresa, proporcionando una funcionalidad coherente, sobre una arquitectura e interfaz comunes.

Este nuevo sistema integrado de información debía llevarse a cabo sobre una plataforma tecnológicamente avanzada.

De acuerdo con esos principios generales, había que alcanzar los siguientes objetivos:

- Tener un único modelo conceptual corporativo que contemplase todas las entidades y reglas de negocio.
- Un modelo de interfaz común a todas las aplicaciones integradas.

- Utilizar los estándares y tecnologías más avanzados.

En 2002 comenzó el diseño del sistema de información técnico-documental, se implementó un modelo de datos único y se migró la lógica de negocio y la base de datos al gestor SQL Server 2000. En 2003 se analizó la arquitectura del sistema y se construyó un framework de desarrollo -constituido por un conjunto de clases genéricas-. Los módulos software y procesos se realizaron en 2004, terminándose el sistema a finales de 2004.

La evolución expuesta da una idea de las distintas etapas que han conducido a Nuclenor a una situación tecnológicamente avanzada con respecto a la gestión de la información.

\section{"Este proyecto integrador del conocimiento técnico-documental ha conducido a Nuclenor a una situación tecnológicamente avanzada"}

\section{Análisis de requerimientos}

La gestión eficiente del conocimiento técnico-documental y la garantía de calidad de la información, son elementos críticos para las empresas del sector nuclear. La utilización de procedimientos, especificaciones de funcionamiento, normativa, guías, manuales y planos, constituyen el soporte de los procesos diarios que realizan este tipo de empresas, y son la piedra angular de la seguridad de las instalaciones.

El enfoque de un proyecto de estas características debe estar alineado con los procesos de negocio de la organización, integrando la gestión de documentos en las formas de trabajo de la empresa (Bustelo, 2006).

La importancia de este hecho llevó a realizar en las fases iniciales del proyecto un profundo análisis de requerimientos, considerando la semántica de las entidades del modelo de conocimiento, así como las funciones y procesos implicados. Para ello se estudiaron las principales herramientas y aplicaciones documentales del mercado, realizando contactos con reconocidas empresas de software documental -Empresarios Agrupados, KEON, etc.- con el propósito de conocer sus productos y enfoques documentales.

En base al conocimiento que se fue recabando se realizó la especificación de requerimientos del sistema, 
considerando una estructura organizada por subsistemas funcionales:
1) de gestión documental,
2) técnicos, $y$
3) de seguridad.

Asimismo, se analizaron las entidades del nivel organizacional -personal, secciones, roles y grupos de trabajo, etc.- y su integración con los subsistemas indicados.

En este trabajo se estudian en profundidad los requerimientos y funcionalidad del subsistema de gestión documental, al constituir éste el núcleo del sistema integrado de información, y en la actualidad un entorno de colaboración para Nuclenor, basado en el ciclo de vida y flujos documentales, y en la utilización de la documentación como soporte de los procesos y decisiones de la empresa.

Los subsistemas técnicos se comentan por el interés que presentan las relaciones que mantienen con la documentación, destacando de qué manera se ha alcanzado la integración del conocimiento técnico y documental.

El modelo de seguridad del sistema se analiza en detalle por tratarse de un área de interés creciente para las organizaciones, especialmente para las empresas del sector nuclear, que trabajan con información sensible y procesos en los que la seguridad y la eficiencia son aspectos críticos.

A continuación se presentan los requerimientos que constituyen la base sobre la que se ha desarrollado el sistema, los cuales proporcionan una visión global de sus capacidades.

\subsection{Gestión documental}

El subsistema de gestión documental recoge los conceptos y la funcionalidad necesaria para realizar el tratamiento electrónico completo de la documentación de la empresa, y tiene por objetivos: 1) implementar el concepto de documentación en el sentido más amplio posible, 2) proveer y gestionar las entidades necesarias para sustituir los archivos físicos tradicionales de la empresa por un archivo electrónico accesible online, 3) proporcionar garantía de calidad a los procesos que requieren el soporte documental 4) favorecer la interrelación entre las entidades técnicas y la documentación, y 5) mejorar la comunicación, distribución y compartición del conocimiento documental en un entorno de colaboración.

\subsubsection{Niveles de abstracción de la documentación}

La documentación es considerada contenido digital almacenado en ficheros con independencia de su formato. El concepto de documento no impone ninguna limitación al tipo de información almacenada, por lo que el sistema puede manejar documentos ofimáticos o digitalizados, planos, imágenes, video, correo electrónico, etc., incluyendo documentos constituidos por más de un archivo vinculado y multiformato.

Asimismo, Nuclenor con objeto de trasladar sus archivos físicos a un archivo electrónico, ha definido como estructura documental una jerarquía de armarios, archivadores, carpetas (subcarpetas) y documentos. Esta estructura ofrece a la organización actualmente la capacidad de acceder y gestionar online la totalidad de sus contenidos documentales.

El archivo electrónico está dividido en dos partes, una primera denominada archivo oficial, que presenta una estructura [armarios, archivadores carpetas (y subcarpetas) y documentos] con un enfoque funcional, y una segunda, denominada archivo particular, que responde a una estructura orgánica por unidades de negocio (secciones) que facilita la clasificación y búsqueda de la documentación a cada sección de la empresa. El archivo oficial es definido y organizado por los documentalistas de la empresa - "gestores de documentación"-, que controlan la seguridad a este nivel, mientras que el archivo particular es gestionado por las distintas secciones que son quienes hacen la clasificación y controlan la seguridad de su documentación estableciendo los permisos de acceso y modificación necesarios.

Los documentos son catalogados de acuerdo con un conjunto de atributos que los caracterizan $-\mathrm{N}^{\mathrm{o}}$ de documento (código de referencia), revisión, fecha del documento (fecha de aprobación), fecha de alta (en el sistema), tipo (procedimiento, plano, especificación, normativa, etc.), subtipo (una clasificación detallada de cada tipo), título, emisor (empresa), estado (válido, histórico, anulado, etc.), soporte (ofimático, ráster, papel), formato, $\mathrm{n}^{\mathrm{o}}$ de hojas, observaciones, fecha y motivo de anulación-, y que son utilizados para su búsqueda relacional y full-text ${ }^{2}$. Este conjunto de atributos han sido definidos por los archiveros de la empresa, de acuerdo con su experiencia en la gestión de los archivos físicos de la organización y en la utilización de aplicaciones documentales como Knosys.

Un documento está constituido por su contenido digital y por una ficha de atributos que lo definen. En un número muy reducido de casos el documento es únicamente la ficha de atributos que lo caracterizan, cuando éste no se almacena electrónicamente, sino que se guarda en formato papel en los archivos físicos de la empresa.

El modelo documental diseñado permite implementar de forma flexible distintas fichas de atributos, de acuerdo con las características y tipología de los documentos que puede interesar a la empresa en cada momento (registro de entradas / salidas de documentos, experiencia operativa, etc.), lo que hace posible la escalabilidad futura del sistema. 


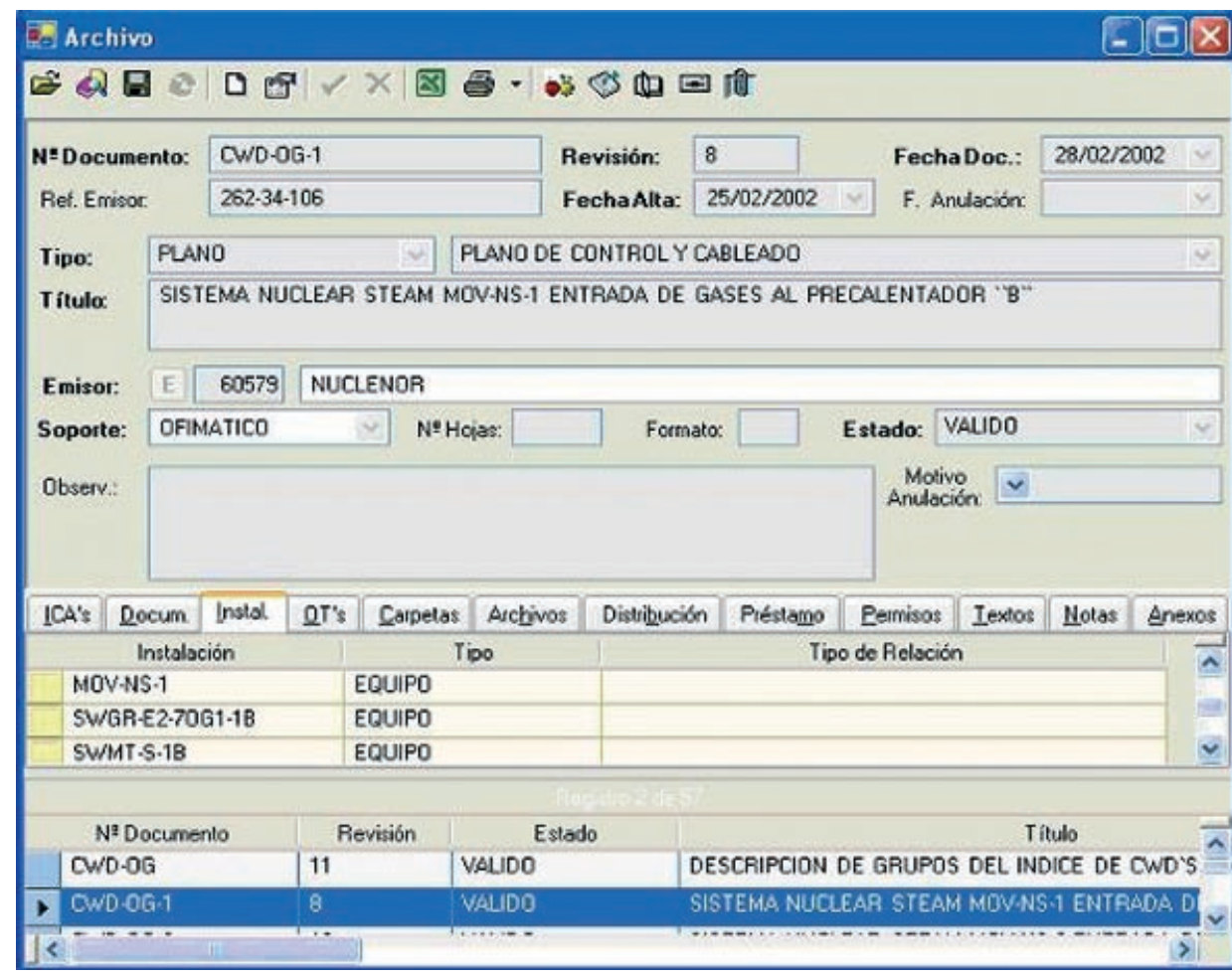

Figura 1. Ficha de catalogación documental
Estas características han contribuido de manera extraordinaria a que el personal deje de considerar la información como un recurso propio, para entenderlo como un activo de la empresa a compartir y mejorar.

La integración del conocimiento técnico y documental ha puesto a disposición de las unidades y del personal de la empresa, de una manera única, toda la información que en ocasiones se encontraba repetida y recogida de distintas formas, en muchos casos incompatibles entre sí, en diferentes aplicaciones. Este hecho ha facilitado un cambio en la cultura de la organización, que ha llevado a unificar la información, a integrar las aplicacio-

No obstante, Nuclenor ha definido una ficha genérica de catalogación y búsqueda de documentos (figura 1) (lo que aporta simplicidad y permite localizar la práctica totalidad de la documentación desde un único punto), una ficha para el registro oficial de documentos enviados y recibidos de otras empresas y organismos a través de su Secretaría, y en el ámbito de los subsistemas técnicos, una ficha para caracterizar los sucesos y experiencias operativas reportadas y documentadas durante la operación de la Planta.

\subsubsection{Trabajo colaborativo}

El subsistema de gestión documental constituye en la actualidad el núcleo de un sistema de colaboración en la empresa. A este hecho han contribuido las siguientes características:

- capacidad del sistema para comunicar mensajes y distribuir documentación entre las personas de la organización de forma sencilla e inmediata;

- disponibilidad de acceso a la documentación en tiempo real, desde el puesto de trabajo, y acceso online al archivo electrónico completo de la empresa;

- garantía de acceso a la documentación vigente, que ha supuesto la desaparición de los archivos propietarios -obsoletos por definición- y un cambio de mentalidad desde la gestión local en cada unidad a una visión de empresa en su conjunto;

- posibilidad de explotar las relaciones de la documentación con las entidades del negocio relevantes: instalaciones-sistemas, equipos, zonas, cables, canalizaciones, etc.-, trabajos de mantenimiento y experiencia operativa. nes, y a trabajar de manera coordinada y colaborativa.

El subsistema de gestión documental permite gestionar el ciclo de vida completo de la documentación -generación, almacenamiento y catalogación, distribución, compartición, utilización y eliminación / conservación- como apoyo a los procesos de negocio y a la toma de decisiones. La participación de distintas unidades en estas fases, ha generado importantes sinergias, principalmente entre las unidades que gestionan la documentación -Administración, Ingeniería, Garantía de Calidad, etc.-, y las unidades que la utilizan. Este hecho ha contribuido a aumentar el grado de interacción entre las secciones, propiciando el trabajo en equipo y la compartición del conocimiento.

Asimismo, la necesidad de proporcionar un entorno de trabajo flexible y de fácil uso, ha llevado a plantear un modelo en el que se han abstraído conceptos de la realidad cotidiana, trasladándolos al ámbito de lo "virtual": la "mesa de trabajo" (figura 2), en la que las personas pueden disponer de los documentos y carpetas con las que están trabajando, las bandejas de entrada y salida que permiten recibir y enviar documentación de forma electrónica, así como la capacidad de poner anotaciones a la documentación, de asociar documentos a cualquier entidad técnica y de relacionar documentos entre sí.

\subsubsection{Archivo electrónico}

La gestión electrónica documental, por las importantes ventajas que ofrece, puede llegar a sustituir a los archivos tradicionales. No obstante, por motivos lega- 
les, organizativos, económicos, etc., puede interesar mantener parte de la documentación en los archivos físicos, y simultáneamente tener acceso electrónico a la misma. Hay que tener en cuenta que en ocasiones no interesa digitalizar la totalidad de los documentos de un archivo físico, dejando en formato papel los documentos históricos cuya frecuencia de consulta es casi nula.

El subsistema de gestión documental, como se ha comentado, permite organizar un

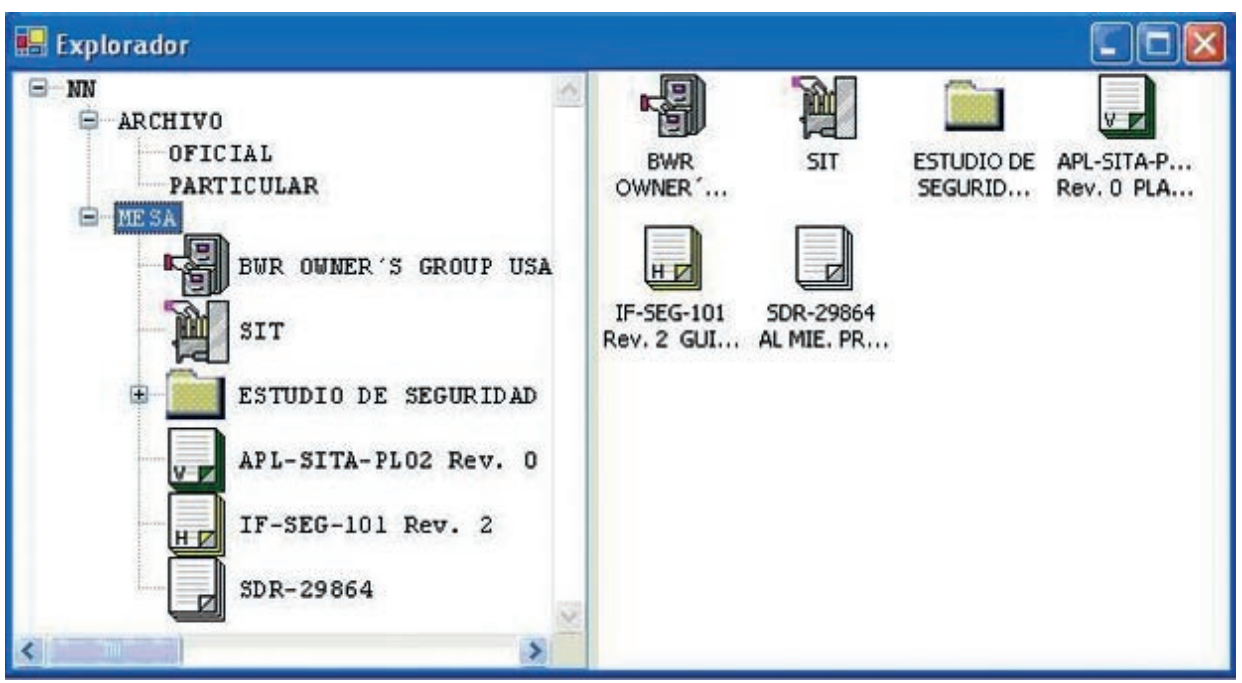

Figura 2: Archivo electrónico archivo electrónico en el que la documentación está estructurada jerárquicamente en armarios, archivadores, carpetas (subcarpetas) y documentos. Asimismo, almacena electrónicamente documentos en cualquier formato y facilita su acceso desde los distintos puestos.

El personal de Nuclenor dispone de acceso a los fondos de la empresa por medio del archivo electrónico corporativo, pudiendo navegar por la estructura jerárquica de dicho archivo virtual hasta localizar la documentación que desea. En otros casos la búsqueda se puede realizar a través de los atributos que caracterizan a los documentos -referencia, título, tipo, estado, etc.-, pudiendo llegar a construir consultas avanzadas, aprovechando la funcionalidad completa del lenguaje relacional SQL -structured query language-.

La figura 2 muestra el archivo electrónico implementado en una ventana de tipo explorador ${ }^{3}$. Este tipo de interfaz es idónea para la presentación y navegación entre las entidades de una estructura jerárquica, y cuando se precisan funcionalidades avanzadas de gestión.

El archivo electrónico es por sí mismo uno de los principales beneficios de la gestión documental en $\mathrm{Nu}$ clenor, por una parte en lo que se refiere al incremento de eficiencia y productividad percibidos, y por otra, en cuanto al ahorro de los costes asociados a los procesos de búsqueda de información.

\subsubsection{Comunicación y distribución de documenta- ción}

La gestión del ciclo de vida de la documentación y la automatización de los flujos documentales, hacen que los sistemas de gestión documental se hayan convertido en una herramienta de gran utilidad en las empresas. Esto es debido a que habitualmente no son las mismas personas las que generan nuevo conocimiento documental, que quienes deben comunicar su existencia y distribuirlo, y en último caso, que quienes lo utilizan para distintos fines. Por esta razón, Nuclenor

consideró la necesidad de poder enviar documentación, comunicar mensajes y dejar constancia oficial de estas acciones cuando fuera preciso.

Para responder a esta necesidad se ha implementado un sistema de comunicación que asegura los niveles de protección establecidos por la organización sobre la documentación, de modo que la responsabilidad de garantizar la seguridad de acceso a la documentación enviada no reside en la persona que la distribuye, sino en el propio sistema de información en aplicación de las limitaciones y directivas impuestas por la empresa. Esta estrategia evita consideraciones de confidencialidad de carácter organizativo y legal.

\section{"El personal ha dejado de considerar la información como un recurso propio, para entenderlo como un activo de la empresa a compartir y mejorar"}

\subsubsection{Control de versiones}

Una adecuada gestión de la documentación requiere controlar las distintas versiones de los documentos, que por su importancia es necesario guardar y poder consultar. Es el caso de los procedimientos de actuación de las empresas del sector nuclear, los planos de las instalaciones que pueden experimentar modificaciones de diseño a lo largo del tiempo, etc.

El sistema técnico-documental de Nuclenor permite gestionar las distintas versiones de la documentación. El sistema conoce el estado en el que se encuentra cada documento: válido, histórico, anulado, etc., de modo que se garantiza el acceso en todo momento a la documentación vigente, salvo petición expresa en sen- 
tido contrario, lo que evita el uso de documentación no actualizada, mejorando la calidad de los procesos y la toma de decisiones.

Una nueva versión de un documento puede ser realizada por cualquier "usuario del subsistema de gestión documental", siempre y cuando tenga permiso de modificación sobre el documento (cada documento tiene definidos sus propios permisos de acceso y modificación). No obstante, las nuevas versiones de documentos oficiales (procedimientos, especificaciones, manuales, etc.) son realizadas por "secretarios", a excepción de las nuevas versiones de planos que son realizadas por "delineantes" de la Oficina Técnica de Ingeniería. Las nuevas versiones de los documentos son puestas en estado válido (oficial) por los "gestores de la documentación”, que revisan su contenido y la catalogación.

\subsubsection{Estrategia de eliminación / conservación de documentos}

La importancia que tiene el soporte documental para los procesos que se realizan diariamente en la empresa, y la necesaria trazabilidad de los procesos de mantenimiento y ejecución de pruebas sobre los equipos de la Planta, hacen que la estrategia a largo plazo sea la conservación de los documentos (se debe tener en cuenta que éstos están asociados a través del sistema con los equipos y trabajos realizados) de manera que pueden pasar con el tiempo a ser históricos o a estar anulados, pero normalmente no son eliminados.

En la operativa diaria los documentos considerados válidos sólo pueden ser eliminados por los "gestores de la documentación", mientras los documentos en preparación (no oficiales) pueden ser eliminados por cualquier usuario con permiso de modificación sobre los mismos.

\subsection{Gestión del conocimiento técnico}

La gestión documental ayuda a mejorar el funcionamiento de las organizaciones. Sin embargo, no es suficiente con almacenar, consultar y compartir la documentación, sino que es necesario poder vincularla con las distintas entidades del negocio (instalaciones, trabajos, etc.), para obtener así el máximo aprovechamiento para la organización.

Consciente de los beneficios de relacionar la información técnica y documental Nuclenor ha definido los mecanismos para integrar estos conocimientos, y específicamente ha considerado las interrelaciones de la documentación con las instalaciones que configuran la estructura de la Central, con los trabajos de mantenimiento y con la experiencia operativa adquirida.

A continuación se describe la funcionalidad de los subsistemas técnicos, y se explica de qué manera se integra la información técnica y documental, analizando las características específicas de cada subsistema a este respecto.

\subsubsection{Gestión de la información técnica y las rela- ciones documentales}

El sistema de información técnico-documental de Nuclenor recoge la gestión de la información técnica a través de un conjunto de subsistemas y módulos específicos que presentan un elevado nivel de integración.

Las relaciones formales con la documentación se establecen fundamentalmente con documentos catalogados, los cuales tienen un carácter oficial dentro de la empresa (figura 1). No obstante, también es posible anexar todo tipo de ficheros (fotografías, archivos de correo, páginas web, documentos de texto, etc.) a las principales entidades (instalaciones, trabajos de mantenimiento, experiencias operativas, etc.) sin necesidad de catalogarlos, y por tanto, evitando una excesiva formalización, en favor de una mayor flexibilidad.

A continuación se explica el propósito de los subsistemas técnicos y las relaciones que mantienen con la documentación, las cuales adquieren su importancia al usar el conocimiento, por cuanto permiten navegar entre la información que se encuentra relacionada.

\section{1) Control de la configuración}

El subsistema de control de la configuración recoge las funciones que permiten gestionar la estructura e instalaciones de la Planta (sistemas, zonas, equipos, cables, etc.).

El objetivo conjunto de los subsistemas de control de la configuración y gestión documental, es mantener coherentes los documentos que reflejan la instalación y los criterios y regulaciones de diseño, con la realidad física y funcional de la Planta. En este sentido el sistema permite relacionar las instalaciones con la documentación (planos, especificaciones, etc.) y anexar ficheros (fotografías, etc.) a las mismas.

Por otra parte, el subsistema de control de la configuración permite la actualización periódica, consulta e impresión de las colecciones de planos, las cuales reflejan la localización, interconexión y estado de los equipos e instalaciones de la Central.

\section{2) Gestión de la experiencia operativa}

El subsistema de gestión de la experiencia operativa permite realizar el tratamiento completo de los hallazgos y sucesos detectados, con el objeto de aprender de los errores propios y de otras centrales mediante un proceso de autoevaluación. Este proceso requiere documentar, analizar y proponer acciones para intentar que los sucesos no se repitan o se minimicen sus consecuencias. Su objetivo es mejorar la seguridad y la fiabilidad de la Central. 
Los sucesos son recogidos y descritos a través de partes de incidencia, los cuales son posteriormente catalogados según una ficha de atributos técnicos específicos (la experiencia operativa), lo que supone el inicio de un flujo de trabajo técnico-documental en el que se evalúa el hallazgo o incidente, y se definen las acciones a tomar para su corrección o mejora, procediendo a continuación a su ejecución.

Las experiencias operativas por sí mismas vinculan el parte de incidente a datos de carácter técnico. Durante la fase de análisis del suceso se pueden establecer relaciones con otros documentos, fundamentalmente con el informe de evaluación del incidente. Además es posible documentar el proceso anexando ficheros a la experiencia operativa (correos electrónicos, formación impartida, documentación de cierre del suceso, etc.).

\section{3) Gestión del mantenimiento}

El mantenimiento de la central de Santa María de Garoña requiere gestionar los trabajos de tipo correctivo y preventivo que es necesario realizar en las instalaciones de la Central.

Los trabajos de tipo correctivo son los que se deben realizar como consecuencia de detectar un mal funcionamiento, el cual puede ser notificado por cualquier empleado mediante la denomina Solicitud de Trabajo (ST).

Para realizar una adecuada gestión de las ST's puede anexarse cualquier tipo de fichero. De este modo el empleado especifica el detalle de la solicitud, incluye una fotografía de la zona de trabajo, indica las pruebas a realizar, etc.

Los trabajos destinados a la prevención de fallos se realizan de forma periódica y planificada. Debido a este carácter periódico, el Trabajo Programado (TP) se define con antelación estudiando las actividades e instalaciones afectadas, así como el estado en el que se debe encontrar la Planta para su ejecución.

Por último, la realización efectiva de los trabajos de mantenimiento se hace con la Orden de Trabajo (OT), que describe con detalle las actividades sobre las instalaciones, adjuntando la documentación necesaria para que el operario complete el trabajo. Tanto las ST's como los TP's se ejecutan a través de una o varias órdenes de trabajo dependiendo del alcance del mismo.

Las tareas programadas y órdenes de mantenimiento requieren del soporte documental necesario, el cual es proporcionado por el sistema que permite asociar cualquier documento, si bien normalmente se vinculan las instrucciones concretas de ejecución del trabajo y los procedimientos de pruebas a realizar.

\subsection{Modelo de seguridad}

Nuclenor ha considerado la seguridad del sistema como un aspecto especialmente importante, y ha dedicado un esfuerzo destacado a la definición de un modelo de seguridad consistente, que contempla los siguientes ámbitos: estructura del sistema de información, seguridad de acceso, gestión de roles, seguridad documental y gestión dinámica de la seguridad.

\subsubsection{Estructura del sistema de información}

La estructura del sistema de información está definida en la capa de datos, que recoge una descomposición del sistema en subsistemas, módulos y funciones. Esta estructura funcional aporta una visión global del negocio y permite gestionar la interfaz principal, denominada ventana marco, que se configura dinámicamente a partir de la información que reside en la base de datos.

Asimismo, la información sobre los ensamblados -objetos software-, clases y métodos que contienen la programación de cada función del sistema, se recoge en la base de datos, de forma que la ventana marco muestra las funciones (si el usuario tiene permiso sobre el método correspondiente), y permite instanciar y ejecutar dinámicamente - utilizando la técnica Reflection ${ }^{4}$ - el método que tiene asignado cada función.

Este conjunto de características permite realizar modificaciones en el sistema con un coste bajo, ya que su estructura y funcionalidad se pueden configurar desde la capa de datos, evitando la necesidad de generar a cada cambio una nueva versión del software.

\subsubsection{Seguridad de acceso}

El valor del conocimiento almacenado y la importancia de los procesos que se pueden llevar a cabo desde el sistema de información, justifica que la organización limite el acceso al mismo. La seguridad a este nivel se controla requiriendo la identificación de la persona por medio de login y contraseña. No se permite utilizar cuentas de usuario genéricas, sino que éstas deben estar vinculadas a una persona reconocida por la organización.

La autentificación en el acceso al sistema es un método eficaz de protección, no obstante Nuclenor ha considerado los inconvenientes derivados de la proliferación de múltiples claves de acceso a los distintos sistemas y aplicaciones de la empresa. Por sí mismo este sistema integrado reduce significativamente la existencia de distintas claves de acceso a aplicaciones que hasta ahora eran independientes.

Hay que tener en cuenta que una parte de los usuarios trabaja desde las propias instalaciones de la organización, y por lo tanto, dentro de sus dominios de confianza. Por otra parte, el propio sistema operativo requiere una autentificación de acceso a la red de la organización.

Por ello se ha considerado un sistema mixto de autentificación que incluye la concepción tradicional, y 
que contempla a su vez la autentificación con clave unificada -single sign on (SSO)-, que permite acceder al sistema sin requerimientos adicionales de validación, más allá de los que impone el acceso a la propia red de la organización.

\subsubsection{Gestión de roles}

Tanto el personal de la empresa como el personal externo contratado, desempeñan distintos roles de trabajo para el desarrollo de su actividad, hecho que queda recogido en el modelo de seguridad.

El rol de usuario para cada subsistema proporciona el acceso a las principales funciones del mismo. A partir de este perfil básico se define un conjunto de roles específicos. La responsabilidad del sistema es compartida por los distintos roles: "gestores de la documentación", "secretarios" (responsables del control de versiones de la documentación oficial: procedimientos, etc.), "gestores de oficina técnica de ingeniería" (responsables de la información técnica de instalaciones, planos y estructura de la Planta), "gestores de mantenimiento" (responsables de los trabajos de mantenimiento) y "coordinadores de experiencias operativas" (responsables del flujo de la experiencia operativa: detección de incidentes, análisis, definición y ejecución de acciones para su corrección).

El modelo de datos contempla las entidades que permiten definir dichos roles de trabajo en la empresa, definir qué funciones puede realizar cada perfil, y asignar aquellos que cada persona necesita. No obstante, también es posible dar autorización de forma individual a los usuarios para realizar determinadas funciones.

La gestión de la seguridad a este nivel se realiza dinámicamente, sin necesidad de hacer costosas modificaciones en los módulos software. La seguridad está definida en un único punto -en la capa de datos- y no se encuentra embebida en los componentes programados.

\subsubsection{Seguridad documental}

La seguridad en la gestión de la documentación corporativa debe considerar tanto los aspectos relativos a la estrategia de almacenamiento, como los que se refieren a su uso controlado. Estas dos perspectivas se comentan a continuación.

\subsubsection{Estrategia de almacenamiento de la docu- mentación}

Constituye un aspecto relevante de la seguridad de la documentación. Las principales estrategias a tener en cuenta son las siguientes:

a) Almacenar los documentos en los sistemas de archivos de la empresa. Este planteamiento tiene algunas ventajas:
- menor complejidad técnica del almacenamiento y recuperación de la documentación, y

- mayor facilidad en el tratamiento de archivos vinculados.

Sin embargo, presenta debilidades que afectan a la propia seguridad, derivadas del hecho de garantizar la consistencia entre la seguridad documental definida en la capa de datos del sistema, y los permisos definidos en el sistema de archivos.

b) Almacenar la documentación en la propia base de datos, utilizando campos de tipo $B L O B^{5}$ - binary large objects- para guardar los documentos (ficheros de texto, binarios, etc.). Esta estrategia presenta una mayor complejidad técnica en el almacenamiento y recuperación de la documentación, lo que supone implícitamente una barrera de protección ante accesos no autorizados. Asimismo, ofrece un alto nivel de integridad a la hora de controlar la seguridad de la documentación, dado que ésta es gestionada únicamente en la base de datos, a través del sistema de información, permitiendo realizar procesos transaccionales con la documentación. Por otra parte, no existe la posibilidad de gestionar permisos fuera del control del sistema, como en el caso anterior.

\subsubsection{Control de acceso a la documentación}

Como se sabe, una parte importante del conocimiento que poseen las empresas se encuentra recogido en su documentación. La gestión electrónica de la misma requiere establecer los mecanismos de seguridad que garanticen su acceso controlado.

El modelo de seguridad en el nivel documental permite definir y controlar para cada elemento -archivador, carpeta, documento-, qué personas están autorizadas a acceder a su contenido y alterarlo. Desde el momento en que un documento es generado o incorporado al sistema se establecen sus permisos de visualización (que garantizan el acceso a su contenido) y edición (que permiten alterar su contenido), tomando los que tiene definidos para ello el usuario que genera / incorpora el documento.

Para garantizar este nivel de seguridad se consideran grupos de personas con características similares con respecto al trabajo que realizan sobre la documentación. No obstante, también es posible asignar autorizaciones específicas (sobre un determinado documento, carpeta, archivador, etc.) a usuarios concretos.

El sistema garantiza la trazabilidad de las operaciones de visualización y modificación de los documentos, registrando la fecha y hora, el usuario que realiza la acción y la operación realizada. La posibilidad de auditar estas acciones permite a la empresa establecer políticas rigurosas de control de acceso a los documen- 
tos y detectar fallos en la seguridad. El administrador del sistema controla el registro de operaciones y puede inhabilitarlo.

\subsubsection{Gestión dinámica de la seguridad}

En la medida que la organización cambia, los sistemas de información deben adaptar su funcionalidad a los nuevos requerimientos. Este tipo de cambios no son hechos aislados, por el contrario los sistemas de información están en constante adaptación.

Es habitual tener que añadir nuevas funciones, y con frecuencia es preciso modificar los parámetros de seguridad que las aplican -definir nuevos elementos de seguridad, dar autorización a las personas y roles de la organización para que puedan realizar determinadas tareas, trabajar de un modo específico, etc.-.

Los costes de adaptación de los sistemas de información a los cambios en la seguridad son relevantes y no son únicamente los directamente cuantificables y derivados del propio desarrollo de las modificaciones. También deben ser consideradas las debilidades implícitas para las organizaciones provenientes de una deficiente política de seguridad de sus sistemas, costes que resultan más difíciles de cuantificar.

En este sentido, las soluciones de seguridad locales y la falta de estrategias de carácter general, debilitan la seguridad de la organización e incrementan notablemente los costes de mantenimiento y adaptación de los sistemas. En otras ocasiones los problemas tienen su origen en la implementación de controles de seguridad en las capas de presentación y de negocio, lo que provoca que los sistemas sean vulnerables ante actuaciones llevadas a cabo directamente en el gestor de base de datos, cuando no se accede a través de las aplicaciones. Además, este tipo de estrategia puede suponer una debilidad adicional para la organización, si se delega la seguridad en los programadores de aplicaciones.

Para el desarrollo del sistema técnico-documental de Nuclenor se planteó una estrategia de seguridad balanceada hacia la capa de datos. El uso de procedimientos ${ }^{6} \mathrm{y}$ funciones almacenadas en el gestor de base de datos, y el empleo de disparadores de lógica ${ }^{7}-$ trig- $^{-}$ gers - han permitido evitar los problemas comentados.

En la implantación de esta estrategia de seguridad toman una importancia destacada las entidades del modelo que permiten definir los elementos de seguridad del sistema, a través de los cuales se configura dinámicamente el comportamiento específico de cada función. Cambios que pueden ser realizados con un reducido coste sobre la base de datos, sin necesidad de modificar los módulos software.

\section{"La seguridad está definida en un único punto -en la capa de datos- y no se encuentra embebida en los componentes programados"}

\section{Modelo tecnológico}

El sistema integrado de información técnico-documental de Nuclenor -en línea con la estrategia y objetivos definidos por la empresa- se ha desarrollado sobre un modelo tecnológico cuyas principales bases son: una arquitectura multinivel, un modelo integrado de datos único, un marco orientado a objetos, una interfaz uniforme y común para todo el sistema, y tecnologías de última generación. A continuación se explican los distintos elementos que constituyen el modelo tecnológico del sistema.

\subsection{Arquitectura del sistema}

El diseño de una arquitectura común para el sistema constituye el soporte fundamental. Es un modelo cliente / servidor de tres capas (figura 3) -datos, negocio y presentación-, en el que la funcionalidad se distribuye entre el nivel de almacenamiento, el nivel de lógica y el nivel de presentación o interfaz. De este modo la capacidad de procesamiento queda repartida entre el servidor y los clientes, lo que permite alcanzar altos niveles de eficiencia. Esta arquitectura multinivel ha permitido realizar una programación por capas, lo que ha proporcionado independencia entre los distintos niveles y una mayor escalabilidad del sistema.

\subsubsection{Capa de datos}

El modelo planteado implementa la capa de datos en un gestor de base de datos único, $-S Q L$ server 2000-. El acceso a los datos se realiza a través de $A D O$ .NET-ActiveX® data objects-. Esta tecnología no depende de conexiones permanentes con la base de datos. Se trata de un modelo desconectado, lo que repercute en un mayor rendimiento de las aplicaciones.

El sistema está disponible en todo momento (24x7), para lo cual el gestor de base de datos se ha instalado con una configuración en cluster, formada por dos equipos Windows 2003 server. La información en el cluster se almacena en un conjunto de discos configurados en Raid 1 y Raid 5 (redundant array of independent / inexpensive disks) ${ }^{8}$, lo que proporciona una gran disponibilidad.

\subsubsection{Capa de negocio}

Un segundo servidor ofrece un conjunto de servicios web que encapsulan parte de la lógica de negocio. 


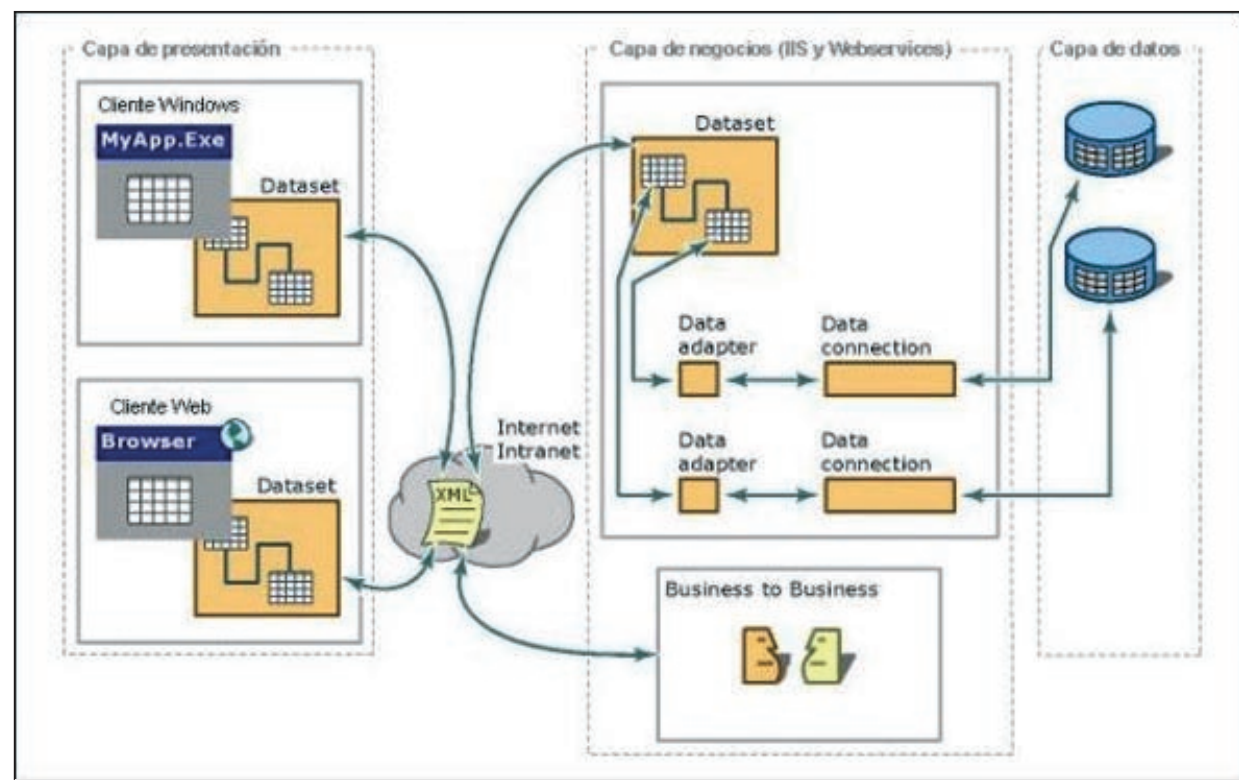

Figura 3: Modelo cliente / servidor de tres capas (fuente: Microsoft) de este modelo unificado se ha realizado un rediseño de las entidades comunes de los sistemas y aplicaciones que ya existían, lo que ha dado lugar a una base de datos única que recoge de forma integrada la mayor parte del conocimiento técnico y documental de la empresa.

Nuclenor ha definido asimismo las directrices para unificar el modo de implementar la lógica de negocio, los dominios y tipos de datos utilizados, los nombres de las entidades y atributos, el uso de mayúsculas y minúsculas, etc., garantizando un marco

El diálogo entre estos servicios web y el resto de componentes que hacen uso de ellos se establece con xml (eXtended markup language). Este equipo también se utiliza como servidor de páginas activas ASP (active server pages) a los clientes web.

\subsubsection{Capa de presentación}

En la capa de presentación se utilizan clientes Windows que ofrecen una interfaz más rica y facilidad de trabajo, y en contrapartida un mayor coste de instalación y mantenimiento, y clientes web (figura 4) que acceden a las páginas $A S P$ suministradas por el servidor, sin necesidad de ningún tipo de instalación específica.

Los costes de instalación y actualización de los ensamblados -objetos software- que utilizan las aplicaciones Windows de escritorio construidas con el Framework .NET, se han reducido de manera muy importante por el uso de la tecnología No-touch deployment, que permite que las aplicaciones puedan ser descargadas a una memoria cache del disco del equipo cliente -assembly download cache-, instaladas y ejecutadas directamente en la máquina del usuario, sin que se produzca ninguna alteración del registro de Windows ni de los componentes compartidos del sistema.

\subsection{Modelo de datos}

El objetivo de integración del conocimiento se ha alcanzado con el diseño de un modelo de datos único para todo el sistema. Para la definición homogéneo para todo el sistema de información.

\subsection{Framework de desarrollo}

Con el propósito de uniformizar el desarrollo se han construido un conjunto de clases genéricas -denominadas framework- capaces de proporcionar la funcionalidad básica común sobre la cual se han programado los módulos del sistema. Dicho marco se ha construido sobre el Framework .NET v.1.1 proporcionado por Microsoft.

La programación de este framework se ha realizado con C\# .NET, por tratarse de un lenguaje moderno orientado a objetos. El propio marco de programación de la plataforma.NET ha sido escrito por Microsoft casi en su totalidad en $C \#$.

El diseño orientado a objetos del framework supone un ahorro de los recursos necesarios para desarrollar

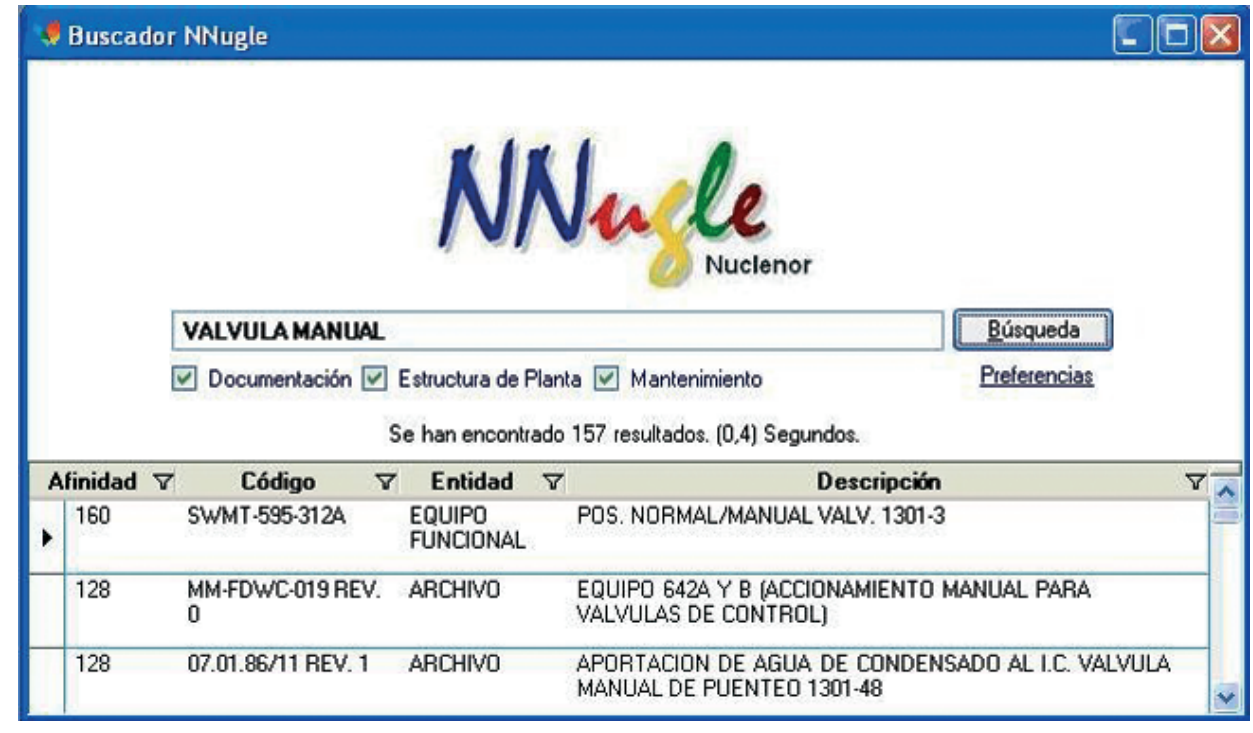

Figura 4: Buscador de información técnico-documental 
los módulos software y amortigua los costes derivados de cambios en el equipo del proyecto. Asimismo, permite desarrollar interfaces de usuario estándar que garantizan un menor esfuerzo de aprendizaje para los usuarios, y reduce los costes de mantenimiento.

El framework implementa las siguientes funcionalidades:

- Seguridad de acceso. A este nivel se considera la identificación de usuarios centralizada e integrada con la seguridad de la red de Windows -autentificación con clave unificada-. La seguridad de acceso se materializa en un conjunto de clases, que manejan además el control de los permisos que tienen los usuarios sobre los módulos del sistema.

- Conexión a la base de datos. Las clases encapsulan los detalles de la conexión al gestor, el tratamiento de fallos en la conexión y los cambios en la configuración de la capa de datos.

- Lógica de negocio. El framework maneja una parte de las reglas de negocio que quedan de esta manera centralizadas para evitar incoherencias, mejorar la productividad y el mantenimiento. Otra parte de la lógica, reglas y restricciones de la organización se implementan en la capa de datos, a través de procedimientos almacenados $^{6}$ y disparadores ${ }^{7}$-triggers- que son controlados por el gestor.

- Interfaces estándar. El framework proporciona los patrones y la funcionalidad básica para construir los diferentes tipos de interfaces: ventanas estándar, que reflejan las propiedades de una entidad y su información relacionada (figura 1), ventanas de tipo explorador ${ }^{3}$ (figura 2), y grids jerárquicos (figura 5), que ofrecen una gran potencia y flexibilidad, al tiempo que proporcionan facilidad para consultar la información.

Los distintos tipos de interfaces definidas han sido consensuadas con los usuarios del sistema, que han participado activamente en su diseño, lo que ha llevado en el caso de las ventanas estándar a implementar una interfaz más tradicional, al que los usuarios ya estaban acostumbrados. Esta estrategia ha contribuido a alcanzar una mejor aceptación del nuevo sistema, y ha reducido los costes de adaptación y aprendizaje de los usuarios.

- Conexión con aplicaciones externas. El framework permite la exportación de la información y los documentos para su tratamiento con otras aplicaciones. Asimismo, dispone de clases que permiten la comunicación de alertas y la distribución de documentos. Mediante un servidor de correo dedicado, las clases recogen las alertas generadas por el gestor de base de datos de acuerdo con los eventos y condiciones que se producen en cada momento, así como los documentos distribuidos por los usuarios, enviándolos al correo electrónico de los destinatarios. Dichas clases integran el estándar $M A P I^{9}$ (messaging application programming interface) -para establecer la comunicación con el servidor de correo de la organización, Microsoft Exchange 2003-.

\subsection{Tecnologías utilizadas}

Nuclenor ha establecido como uno de los objetivos principales del proyecto la utilización de herramientas abiertas de desarrollo y soporte del conocimiento, basadas en los estándares del mercado, que permitan situar tecnológicamente a la empresa en una posición de vanguardia en la utilización de las tecnologías de la información.

- En el diseño del modelo de datos se ha empleado Sybase PowerDesigner v. 9.5., y como gestor de bases de datos SQL Server 2000 sobre Windows Server 2003.

- La plataforma de desarrollo ha sido Visual studio .NET 2003 Enterprise architect, -el lenguaje de programación $C \#-$.

- El control de la configuración del software se ha realizado con la herramienta Visual SourceSafe v. 6.0.

\section{"El coste de adaptación de los sistemas de información a los cambios en la seguridad puede ser importante"}

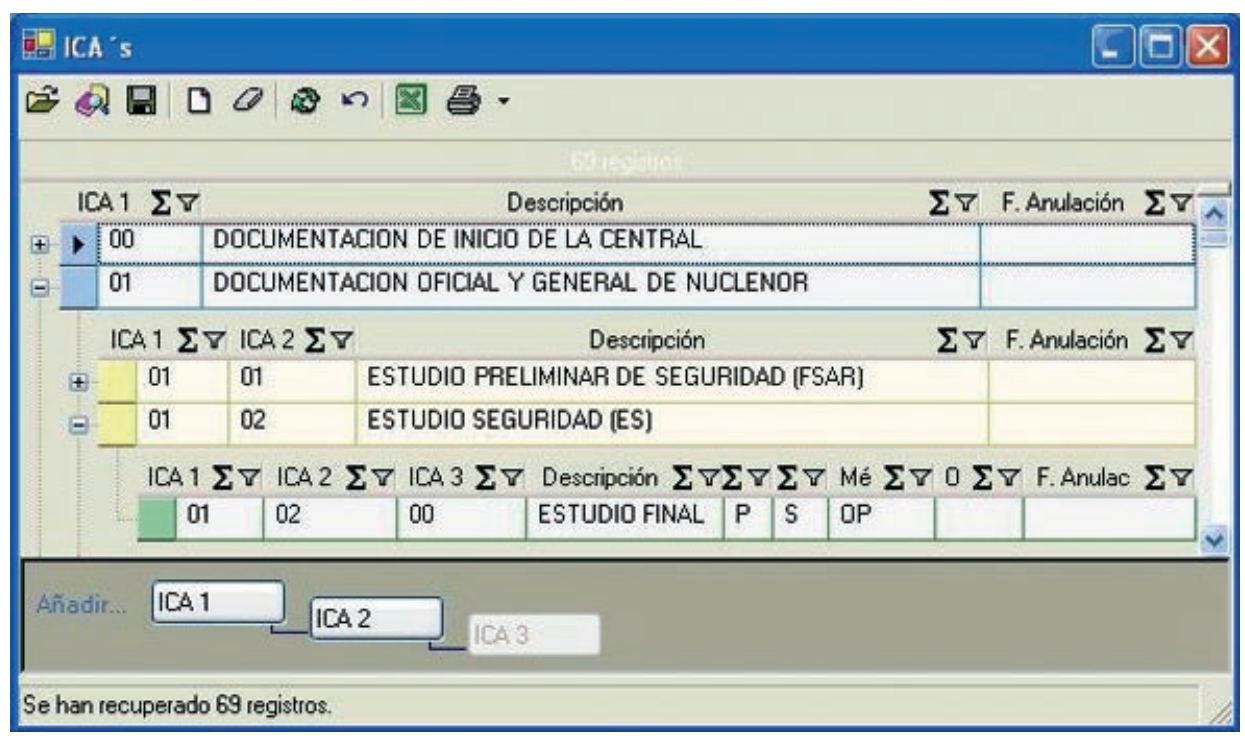

Figura 5: Índice común de archivo (ICA) 


\section{Análisis de resultados}

En el ámbito del conocimiento y sus procesos de gestión, cabe señalar el aumento en la creación de nuevo conocimiento -se generan 5.701 nuevos documentos anuales de media-. Se ha producido el cambio de los procesos basados en el uso de papel a los procesos electrónicos. De hecho el ciclo de vida de la documentación se desarrolla a través del sistema, lo que ha reducido la utilización de documentos en papel, tanto la impresión (los documentos están disponibles online), como la distribución de múltiples copias de documentos que se venía realizando (la distribución actualmente se realiza de forma electrónica). Ha mejorado la eficiencia de los procesos documentales (el tiempo medio de búsqueda de un documento es inferior a 1 minuto), que se realizan actualmente desde el puesto del empleado, que no necesita desplazarse a otro departamento o al archivo para buscar o entregar un documento.

Se están alcanzando altos niveles de colaboración en base al desarrollo de los flujos de trabajo documentales y técnicos, logrando sinergias entre unidades de negocio y empleados. La mejora de la colaboración y coordinación de las actividades tiene su origen en la gestión del conocimiento compartido por toda la organización. Ahora las actuaciones que realizan las distintas unidades sobre la información afectan a las demás y al sistema en su conjunto. Por otra parte, la utilización intensiva de los canales electrónicos para la comunicación de alertas (que avisan de las implicaciones que los cambios en la información pueden tener para otras secciones) y la distribución de documentación -con una media de 65.373 documentos distribuidos anualmente-, están favoreciendo la coordinación y la colaboración.

En opinión de los distintos mandos de cada departamento, la empresa ha mejorado la calidad de la información, a lo que ha contribuido de manera decisiva la integración del conocimiento en un modelo de datos único, que ha eliminado la redundancia de la información y ha permitido implantar procesos consistentes. El conocimiento técnico-documental es compartido ahora por toda la organización, existe por tanto una mayor visibilidad de la información, lo que facilita su continua actualización y mejora. El control de versiones de la documentación y la garantía de acceso a la documentación vigente están contribuyendo asimismo a desarrollar una cultura de calidad.

En el ámbito de la seguridad, los empleados realizan las actividades y procesos que constituyen su trabajo según las autorizaciones establecidas por la empresa, las cuales pueden ser actualizadas en cualquier momento sin costes adicionales de programación, debido a que la seguridad se encuentra definida en la capa de datos. El conocimiento documental está sometido a los niveles de confidencialidad definidos por la organización, los documentos asociados a determinadas temáticas tienen restringido el acceso a las personas que la empresa ha establecido. Por otra parte, la distribución electrónica de documentos permite, cuando es necesario, garantizar el acceso exclusivo al personal que recibe dicha documentación.

El sistema está contribuyendo a desarrollar de forma electrónica la "memoria organizacional" (Ackerman y Halverson, 2000), ya que se ha capturado e institucionalizado una gran parte del conocimiento de la empresa, contribuyendo a incrementar el capital estructural de la organización. La memoria organizacional es el centro del sistema de gestión del conocimiento (Meroño, 2004). Los procesos de aprendizaje han mejorado debido a la utilización de una interfaz común, lo que ha reducido los costes de formación de los usuarios, que en la actualidad son capaces de asumir la mayor parte de las modificaciones y nuevos desarrollos sin necesidad de formación adicional.

La medición de los resultados es realizada por la sección de Garantía de Calidad que evalúa periódicamente los parámetros de calidad del sistema y realiza el seguimiento de distintos indicadores: documentación generada, relaciones entre la documentación y las instalaciones de la Central, experiencias operativas y hallazgos reportados, etc. No obstante, las distintas secciones responsables del conocimiento, evalúan permanentemente el sistema y su contribución a la mejora de la calidad y gestión de la información.

\section{"Un conjunto de clases genéricas -denominadas framework- proporcionan la funcionalidad básica común sobre la que se han programado los módulos del sistema"}

\section{Líneas de investigación}

En la actualidad existen varias líneas de investigación abiertas basadas en este núcleo de gestión de conocimiento. En primer lugar, se están innovando los procesos administrativos, e incorporando este nuevo conocimiento al sistema. En segundo lugar, se están automatizando parte de los procesos y formas de trabajo actuales, de manera que puedan ser realizados de forma desatendida por agentes software. Por último, se están implementando servicios avanzados de gestión de la información; es el caso del buscador corporativo de información denominado NNugle (figura 4), que 
mediante un motor de búsqueda full-text ${ }^{2}$ actúa sobre la base de conocimiento, incluyendo los contenidos documentales y la información técnica.

\section{Conclusiones}

La implantación de sistemas de gestión de información técnico-documental requiere utilizar de forma intensiva las tecnologías de la información, aplicar técnicas específicas para el tratamiento de documentos (procedimientos, planos, imágenes, etc.), y combinar múltiples herramientas de trabajo -digitalización y tratamiento de imágenes, procesamiento de textos, delineación de planos, etc.-. Por otra parte, es necesario integrar el conocimiento disperso que se encuentra en distintas fuentes de información -bases de datos, archivos documentales, información técnica, flujos de trabajo y experiencia de las personas-. Este conocimiento, en algunos casos no explícito, debe ser institucionalizado e incorporado al capital estructural de la organización.

La implicación de la dirección y del personal de la organización son factores clave para el éxito de este tipo de proyectos, debido a que suponen una transformación significativa de las formas de trabajo. Asimismo resulta particularmente importante hacer ver a los empleados las ventajas que traerá el sistema para su trabajo cotidiano.

La implantación de un sistema integrado de gestión de información técnico-documental en Nuclenor ha provocado un cambio sustancial en las formas de trabajo y operativa de los empleados, que ahora utilizan el sistema a diario para realizar sus tareas, y ha contribuido notablemente a mejorar la cultura de calidad en la empresa. Las sinergias alcanzadas entre las unidades y el personal que participa de forma colaborativa en los procesos técnicos y flujos documentales, están favoreciendo la coordinación y la mejora continua en la organización.

La disponibilidad online de documentos e información técnica, y las interrelaciones entre ambos tipos de conocimiento, contribuyen notablemente a mejorar la productividad. Asimismo, la integración de conocimientos, su actualización permanente y la garantía de acceso a la documentación vigente, reduce la posibilidad de cometer errores, aspecto especialmente importante en la industria nuclear.

La utilización de tecnologías avanzadas como $R e$ flection ${ }^{4}$ y No-touch deployment, así como la gestión y configuración de la estructura, funcionalidad y seguridad del sistema de información desde la capa de datos, permiten realizar modificaciones de forma dinámica, reduciendo los costes de mantenimiento y evitando la necesidad de generar en cada cambio una nueva versión del software.
Este tipo de sistemas fomenta que el conocimiento resida en la empresa y no en las personas, facilita el rediseño e innovación de los procesos de la organización, y estimula la colaboración y la compartición del conocimiento.

Agradecimientos: este trabajo ha sido posible gracias al apoyo continuado a la investigación y a la financiación prestada por la empresa Nuclenor $S A$, así como al soporte proporcionado por la Fundación Leonardo Torres Quevedo (FLTQ) a la gestión de la investigación.

\section{Notas}

1. El conocimiento explícito - explicit knowledge- es el conocimiento individual y colectivo que ha sido codificado típicamente como objetos, palabras y números, en forma de gráficos, figuras, especificaciones, manuales, procedimientos, etc., y que puede ser comprendido y compartido fácilmente (CWA 14924-5, 2004).

2. Las búsquedas por texto integro -full-text- permiten realizar consultas por todo el contenido de documentos y campos de las bases de datos. Se utilizan habitualmente operadores de cercanía -“near"-, siendo posible localizar las palabras derivadas, frases completas, utilizar operadores "and", "or" y "not", y obtener el ranking o grado de relevancia con que los resultados se adaptan a los criterios de búsqueda.

3. La ventana de tipo explorador responde a una interfaz con características y funcionamiento similares al explorador de Windows, si bien se trata de un desarrollo propio que permite su adaptación a distintos contextos -en particular este tipo de ventana ha sido utilizada para el entorno y las funciones documentales del sistema-.

4. Reflection es la técnica que presenta la plataforma .NET para tratar objetos en tiempo de ejecución. Permite inspeccionar los objetos, obtener los elementos que los componen (eventos, métodos, propiedades, etc.) y trabajar de manera dinámica con ellos.

5. BLOB -binary large objects-, término que hace referencia a campos de bases de datos que permiten almacenar en formato binario objetos de gran tamaño. Generalmente estos datos son imágenes, archivos de sonido y objetos multimedia, aunque también pueden almacenar ficheros en cualquier formato.

6. Un procedimiento almacenado-stored procedure-es un programa que se encuentra localizado físicamente en una base de datos. Su principal ventaja es que se ejecuta directamente en el motor de bases de datos, y por lo tanto de forma muy eficiente, ya que tiene acceso directo a los datos. Asimismo su utilización ofrece ventajas cuando una base de datos es manipulada desde distintos programas externos. Embeber la lógica de la aplicación en la base de datos, evita incluir la misma lógica en todos los programas que acceden a los datos, lo que simplifica la creación y el mantenimiento de los programas involucrados.

7. Un disparador -trigger- es un evento asociado a las operaciones de inserción, actualización y borrado de las filas de una tabla, que provoca que el gestor de base de datos ejecute una lógica programada en respuesta a dicha operación. Los disparadores son utilizados para automatizar procesos y controlar reglas de negocio.

8. El término RAID -redundant array of independent / inexpensive dis$k s$ - hace referencia al conjunto de discos redundantes independientes y económicos que se utilizan para aumentar la integridad de los datos en los discos, mejorar la tolerancia a fallos y el rendimiento. Los sistemas RAID se implementan en 7 configuraciones o niveles: RAID 0 a RAID 6 y existen también combinaciones de niveles de RAID. A nivel práctico sólo los RAID impares, junto a las combinaciones de estos, se han impuesto en el mercado: RAID $1,3,5,1+0$, y $0+1$, destacando por su aceptación sobre los demás el RAID $1,5,1+0$, y $0+1$.

9. MAPI -messaging application programming interface-, hace referencia al interface de programación que permite que una aplicación envíe y reciba correo a través del sistema de mensajería Microsoft Mail. 


\section{Bibliografía}

Ackerman, M.; Halverson, C. "Re-examining organizational memory". En: Communications of the ACM, 2000, v. 43, n. 1, pp. 58-63.

Alòs-Moner, A. "Gestión documental: aspectos previos a su implementación”. En: El profesional de la información, 2006, v. 15, n. 3, pp. 222 226.

Bustelo, C. "Gestión de documentos: enfoque en las organizaciones". En El profesional de la información, 2006, v. 15, n. 1, pp. 4-5.

Barranco, A. "Gestión documental. Un nuevo recurso estratégico". En: $L a$ vanguardia, 27-05-2006, pp. 3.

CWA 14924-5. "European guide to good practice in knowledge management. Part 5: KM terminology". En: Comité Européen de Normalisation (CEN), 2004, pp. 1-29.
Marwick, A. "Knowledge management technology". En: IBM systems journal, 2001, v. 40, n. 4, pp. 814-830.

Meroño, A. L. "Tecnologías de información y gestión del conocimiento: integración en un sistema”. En: Economía industrial, 2004, n. 357, pp. $107-$ 116.

Pedro Solana-González y Daniel Pérez-González, Facultad de Ciencias Económicas y Empresariales, Univ. de Cantabria, Av. los Castros, s/n. 39005 Santander, España.

pedro.solana@unican.es

daniel.perez@unican.es

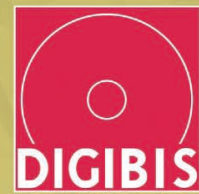

\section{Definimos espacios virtuales} avanzados para la gestión del conocimiento en la Web y la preservación digitol a largo plazo

Herramientas para crear espacios virtuales

DICHARCH 1.6

Sistema digital de descripción y gestión archivística

DICIBIB 4.0

Solución avanzada para la creación de Bibliotecas Digitales y la Gestión Bibliotecaria Multilingüe

Digifalizeción avenzreda Con asignación dinámica de metadatos

\section{OAsls-PMH 2.0}

Sistema integrado de recolección de diversos esquemas de metadatos.

DCMI sin cualificar

MARC 21

EAD

mod_OAl

ePrints (diversos esquemas)

Ponderación de repositorios

\section{ORACLE" \\ PARTNERNETWORK}
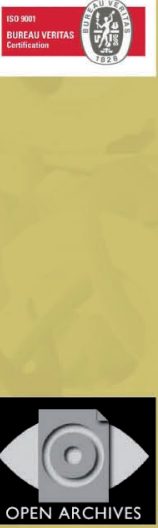

Validación en el Data Providers de la Open Archives Intiative.

SiteMaps con Google y Yahoo.

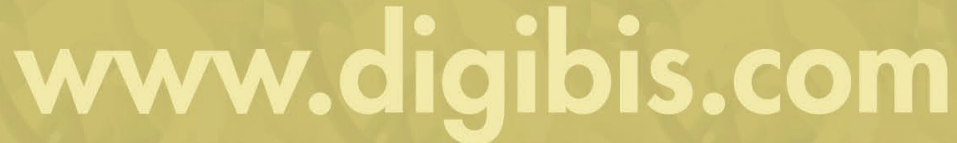

- Recolección en la Web para Entidades e Instituciones de Memoria en OAI-PMH y Dublin Core e intercambio de metadatos en METS (diferentes Profiles)

- Consultoría y mappings a DCMI para implementación en repositorios OAl v2.0

- Tecnologías abiertas para la creación, recuperación y recolección de metadatos y anotación de instancias (MARCXML, DCMI y RDF)

- Repositorios Institucionales para Preservación Digital a largo plazo mediante PREMIS y OAIS ISO 14721 